Research Article

\title{
Time-Domain Analysis of Coupled Carbon Nanotube Interconnects
}

\author{
Davood Fathi \\ School of Electrical \& Computer Engineering, Tarbiat Modares University (TMU), P.O. Box 14115-194, Tehran, Iran \\ Correspondence should be addressed to Davood Fathi; d.fathi@modares.ac.ir
}

Received 29 December 2013; Accepted 5 March 2014; Published 4 May 2014

Academic Editor: Carlos R. Cabrera

Copyright (C) 2014 Davood Fathi. This is an open access article distributed under the Creative Commons Attribution License, which permits unrestricted use, distribution, and reproduction in any medium, provided the original work is properly cited.

\begin{abstract}
This paper describes a new method for the analysis of coupling effects including the crosstalk effects between two driven coupled single-walled carbon nanotubes (SWCNTs) and the intertalk effects between two neighboring shells in a multiwalled carbon nanotube (MWCNT), based on transmission line circuit modeling. Using rigorous calculations, a new parametric transfer function has been obtained for the analysis of the impact of aggressor line on the victim line, which depends on the various coupling parameters such as the mutual inductance, the coupling capacitance, and the tunneling resistance. The influences of various parameters such as the contact resistance and the switching factor on the time behavior of coupling effects between the two coupled CNTs and an important effect named "crosstalk-induced delay" are studied and analyzed.
\end{abstract}

\section{Introduction}

Carbon nanotubes (CNTs) have owned a major importance for the next generation nanoscale technologies, since their discovery [1]. CNTs exhibit a ballistic flow of electrons with electron mean free paths of several micrometers and are capable of conducting very large current densities [1-3]. They are therefore proposed as potential candidates for signal and power interconnection $[4,5]$. Depending on their chirality (the direction along which the graphene sheets are rolled up), CNTs demonstrate either metallic or semiconducting properties. Carbon nanotubes are also classified into singlewalled (SWCNT) and multiwalled nanotube (MWCNT). Figure 1 shows the equivalent distributed circuit model of an individual CNT (shell in a MWCNT). In this figure, $R_{\mathrm{mc}}$ is the imperfect contact resistance, $R_{\mathrm{Q}}$ is the quantum resistance, $R_{S}$ is the scattering-induced resistance, $L_{K}$ and $L_{M}$ are the kinetic and magnetic inductances, respectively, and $C_{\mathrm{Q}}$ and $C_{E}$ are the quantum and electrostatic capacitances, respectively.

The quantum resistance is equally divided between the two contacts on either side of the nanotube and can be expressed as $R_{F}=h /\left(4 e^{2}\right)$ [6] where $h$ is plank's constant and $e$ is electron charge. For lengths greater than the mean free path value $\left(\ell>\lambda_{\mathrm{CNT}}\right)$, scattering leads to an additional ohmic resistance which increases with length as $R_{S}=R_{F}\left(\ell / \lambda_{\mathrm{CNT}}\right)$ [7]. The total capacitance of a CNT arises from two sources: the electrostatic capacitance which is the intrinsic plate capacitance of an isolated CNT and the quantum capacitance which accounts for the quantum electrostatic energy stored in the nanotube when it carries current $[8,9]$. Their relations can be found in $[4,8-11]$. The total inductance of a CNT arises from two sources: the magnetic inductance and the kinetic inductance. Their relations can be found in [10-12].

The value of mean free path (MFP) for a CNT is diameter dependent and irrespective of the nature of SWCNTs (shells in an MWCNT), metallic or semiconducting, and we can assume $\lambda_{\mathrm{CNT}} \approx 1000 D$ [13].

Because of the high intrinsic resistance of a SWCNT, CNTs are used generally as CNT-bundle or multiwalled carbon nanotube (MWCNT) [3, 13-15]. Since in a bundle, all CNTs are connected to the same driving voltage and also they have equal geometric characteristics, the crosstalk effect between different CNTs in the bundle is not important. In a MWCNT, each shell, which itself is a SWCNT, has different geometric characteristics from the other shells. Also the contact resistance of each shell depends on its diameter $[14,16]$ and therefore is different from the other shell. The two mentioned differences between shells of a MWCNT imply that the distribution of potential through different shells 


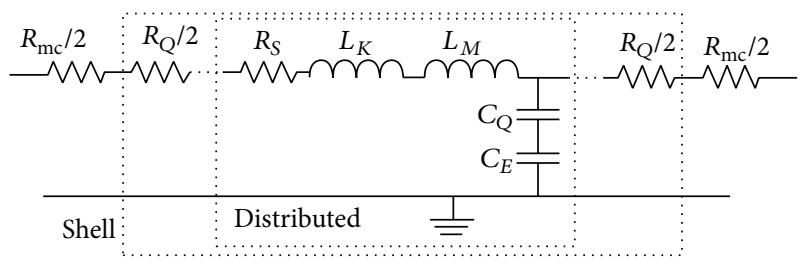

FIGURE 1: Equivalent distributed circuit model of an individual CNT (shell in a MWCNT) [13].

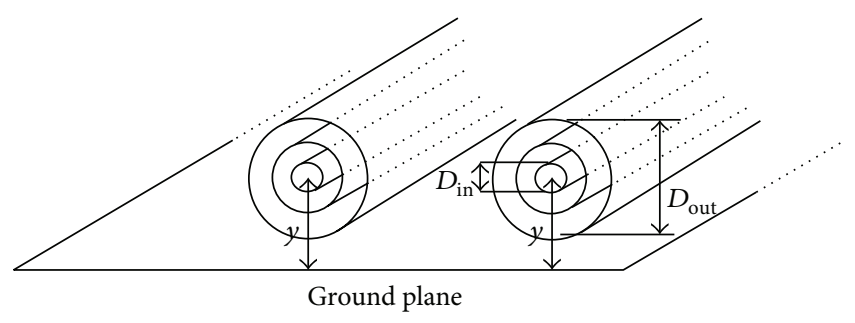

FIgURe 2: Two coupled MWCNTs over a ground plane.

is not similar and this fact causes the coupling (intertalk) effects between neighboring shells to have a major role. On the other hand, many of MWCNTs are often placed together in a VLSI circuit in order to achieve the high interconnect performances. Therefore, the coupling between the two adjacent MWCNTs would be very important. On the other hand, since the input driving voltage applied to each MWCNT might be different from each other, the analysis of coupling effects between them in the latter configuration would own a major importance.

\section{The Coupling Effects between Two Coupled SWCNTs (Two Shells in a MWCNT)}

Figure 2 shows two neighboring MWCNTs which their outer shells affect each other and therefore the coupling between them should be considered.

As it is clear from Figure 2, the neighboring shells in a MWCNT or the outer shells of two adjacent MWCNTs affect each other. The related interactions depend on the coupling capacitance, the mutual inductance, and the tunneling resistance, which will be discussed in the subsequent section.

2.1. The Coupling Effect Parameters. In this section, the various coupling effect parameters in CNTs are defined and described. These parameters are classified into two categories. One category which is related to the interactions between the inner two neighboring shells in a MWCNT is named "intertalk effect" parameters and the other which is related to the interactions between the two coupled SWCNTs (or the outer shells of two adjacent MWCNTs) is named "crosstalk effect" parameters.
2.1.1. The Coupling Capacitance. The coupling capacitance between two parallel carbon nanotubes each of length $\ell$ is given by $[11,17]$

$$
C_{C}=\frac{\varepsilon \pi \ell}{\ln \left(s / D+\sqrt{(s / D)^{2}+1}\right)},
$$

where $s$ and $D$ are the center-to-center spacing between the two CNTs and the diameter of each CNT, respectively. Also the shell-to-shell coupling capacitance per unit length in a MWCNT can be obtained by using the coaxial capacitance formula [13]

$$
C_{S}=\frac{2 \pi \varepsilon}{\ln \left(D_{\text {out }} / D_{\text {in }}\right)},
$$

where $D_{\text {in }}$ and $D_{\text {out }}$ are the diameters of the inner and outer shells, respectively. It should be noted that (2) is for the two neighboring shells in a MWCNT, where the space between them is $0.34 \mathrm{~nm}[13,18]$, and therefore we have $D_{\text {out }}-D_{\text {in }}=$ $0.34 \mathrm{~nm}$.

2.1.2. The Tunneling Resistance. The tunneling conductance through the $\pi$ orbital overlap between two atoms of nearby shells in a multiwalled CNT (MWCNT) or a single-walled CNT (SWCNT), assuming elastic tunneling, is given by [19]

$$
G_{T, \text { atom }}=\frac{4 \pi e^{2}}{\hbar} N_{\text {atom }}^{2} E_{\text {bin }}^{2},
$$

where $E_{\text {bin }}$ is the binding energy due to electronic delocalization and

$$
N_{\text {atom }}=\frac{2 n}{h v_{F}} \frac{S}{2 \pi r}
$$

is the density of state per atom. $S$ is the surface occupied by one atom, that is, $2.6\left(\mathrm{~A}^{\circ}\right)^{2}[19], r$ is the shell (or SWCNT) radius, $v_{F}$ is the Fermi velocity which is approximately $8 \times$ $10^{5} \mathrm{~m} / \mathrm{s}$ [10], and $n$ is the number of modes due to doping which is between 10 and 20 [19]. The binding energy $E_{\text {bin }}$ has been theoretically estimated at $\approx 25 \mathrm{mev}$ and measured around $\approx 35 \mathrm{mev}$ [19]. Assuming $n=15, r=5 \mathrm{~nm}$, and $E_{\text {bin }}=35 \mathrm{mev}$, we obtain $G_{T, \text { atom }} \approx 2.0855 \times 10^{-10} 1 / \Omega$ using (3), (4). The intershell conductance in a MWCNT is related to the tunneling conductance $G_{T, \text { atom }}$ through $g=$ $G_{T, \text { atom }}(2 \pi r / S)$. Using the above mentioned values we obtain that $g \approx(4 \mathrm{k} \Omega)^{-1} / \mu \mathrm{m}$, which is in agreement with the reported values $[19,20]$. Since the coupling area in a SWCNT is approximately the surface of an atom, the cross coupling conductance between the two adjacent CNTs is very small with respect to the above obtained value for the intershell conductance in a MWCNT. In this case, we can express the cross coupling conductance as $g^{\prime}=G_{T, \text { atom }}(2 \pi r / S) / N$, where $N$ is the number of atoms per the cross section of CNT and thus can be neglected in the crosstalk effect analysis between the two coupled adjacent CNTs. This discussion will be described in detail in Section 2.2. 


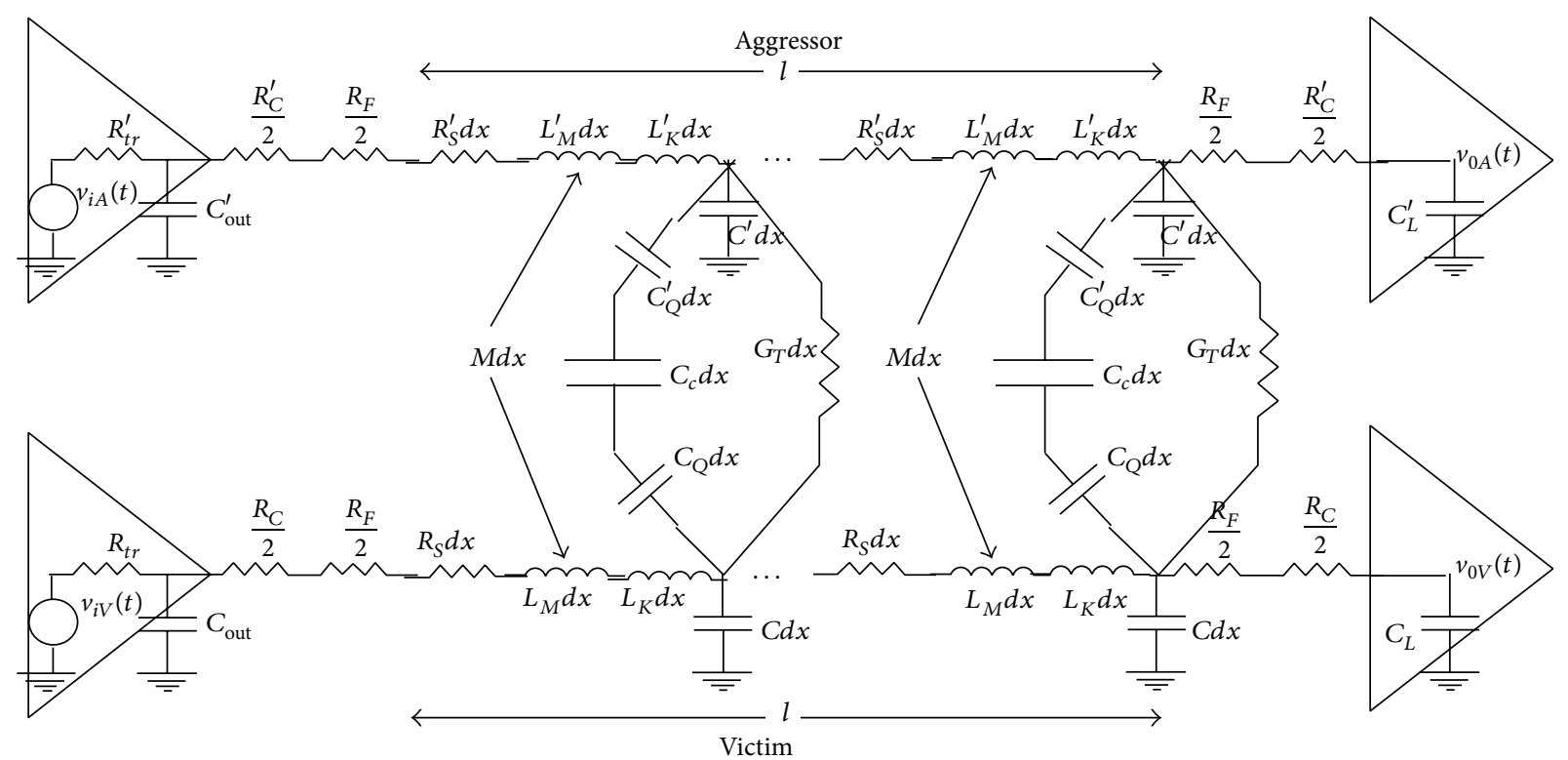

FIGURE 3: Equivalent circuit model for two coupled aggressor-victim CNT interconnects (the two neighboring shells in a MWCNT or the outer shells of two adjacent MWCNTs), with coupling effect parameters.

2.1.3. The Mutual Inductance. The mutual inductance between two parallel current carrying wires can be calculated using [21]

$$
M=2 \ell\left[\ln \left(\frac{\ell}{s}+\sqrt{1+\left(\frac{\ell}{s}\right)^{2}}\right)-\sqrt{1+\left(\frac{s}{\ell}\right)^{2}}+\frac{s}{\ell}\right],
$$

where $M$ is the mutual inductance in $\mathrm{nH}$ and $\ell$ is the wire length in $\mathrm{cm}$. Also $s$ denotes the geometric mean distance between the wires, which is approximately equal to the pitch of wires. As reported in the literature (e.g., [15]), (4) can be used for the calculation of mutual inductance between two coupled CNTs, where $s$ is the center-to-center spacing between the two nanotubes. For the case where $\ell / s \gg 1$, the mutual inductance using (4) is simplified as

$$
M=2 \ell\left[\ln \left(\frac{\ell}{s}\right)-0.3069\right]
$$

The mutual inductance per unit length between different shells in a MWCNT can be derived from [13]

$$
M_{\text {shell }}=\frac{\mu}{2 \pi}\left[\ln \left(\frac{4 \ell}{D_{\text {out }}}\right)-1+\frac{D_{\text {out }}+D_{\text {in }}}{\pi \ell}\right],
$$

where $\ell$ is the length of each shell and $D_{\text {in }}$ and $D_{\text {out }}$ are the diameters of the inner and outer shells, respectively.

2.2. The Coupling Effect Analysis. Figure 3 shows two coupled CNT interconnects each with length $\ell$ where one has been assumed as aggressor line and the other has been considered as victim line. As mentioned in Section 1 and as clear from Figure 2, these two coupled lines (interconnects) might be the two neighboring shells in a MWCNT or the outer shells of two adjacent MWCNTs.

In this figure, $R_{F}$ is the fundamental (quantum) resistance, $R_{S}$ is the scattering resistance per unit length of the victim line, $R_{C}$ is the contact resistance of the victim line (which will be described in Section 3.2), $L_{M}$ and $L_{K}$ are the magnetic inductance and the kinetic inductance per unit length, respectively, of the victim line, and $C$ is the capacitance per unit length of the victim line which consists of the electrostatic per unit length $C_{E}$ and the quantum capacitance per unit length $C_{Q}$. The victim line is driven by a driver with the output resistance and output capacitance of $R_{t r}$ and $C_{\text {out }}$, respectively, and is driving a load capacitance of $C_{L}$. Also $M, C_{C}$, and $G_{T}$ are the mutual inductance, the coupling capacitance, and the tunneling conductance per unit length, between the two CNTs, respectively. These parameters and their relations were mentioned in the previous section. The corresponding mentioned parameters of the aggressor line have been cleared with the prime (I) notation in Figure 3.

It should be noted that, for two similar coupled SWCNTs, the circuit model parameters mentioned above can be assumed the same for the aggressor and victim interconnect lines. Also since the space between the two neighboring shells in a MWCNT is $0.34 \mathrm{~nm}[13,18]$, assuming their diameters very larger than this value implies that we can consider their diameters approximately equal and therefore the circuit model parameters which are diameter dependent can be assumed the same for the aggressor and victim lines.

In the discussion of crosstalk effects between coupled interconnects, an important variable (effect) is defined and analyzed. This effect is "crosstalk-induced delay" which introduces the impact of the input voltage and circuit parameters 


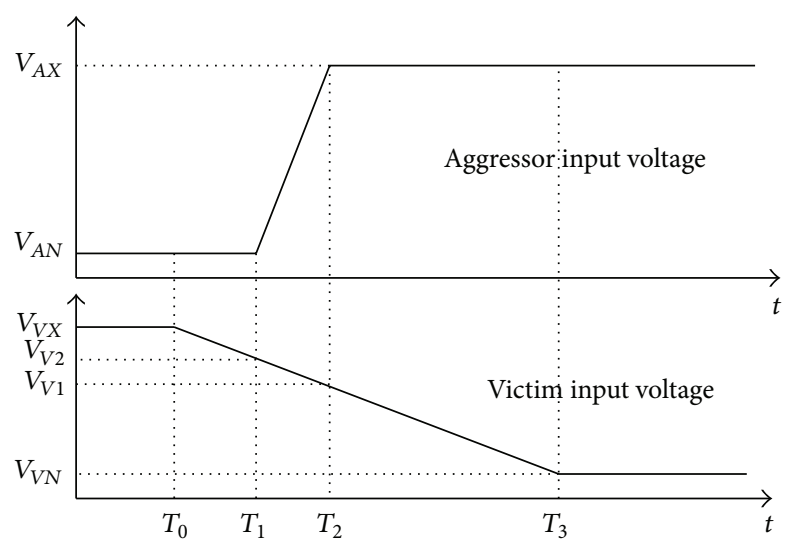

FIGURE 4: Input voltages of the aggressor and victim lines.

of the aggressor line on the delay of the voltage at the end of the victim line. For this purpose, we define the input voltages of the aggressor and victim lines, as shown in Figure 4.

For analyzing the impact of the aggressor line on the victim line in Figure 3, we define $k=K_{1} / K_{2}$ as the switching factor in which $K_{1}$ and $K_{2}$ are the slope of aggressor line input voltage and the slope of victim line input voltage, respectively. In this respect, for the circuit analysis purpose, we consider the equivalent circuit model defined in Figure 5. In this figure, in order to show the impact of the aggressor line on the victim line, the following crosstalk-induced circuit parameters have been defined:

$$
\begin{gathered}
L_{\mathrm{MV}}=L_{M}-k M, \\
G_{\mathrm{TV}}=(1-k) G_{T}, \\
C_{\mathrm{CV}}=\frac{(1-k)}{1 / C_{C}+2 / C_{\mathrm{Q}}},
\end{gathered}
$$

where $L_{\mathrm{MV}}, G_{\mathrm{TV}}$, and $C_{\mathrm{CV}}$ are the total equivalent magnetic inductance of the victim line, the total equivalent tunneling conductance, and the total equivalent coupling capacitance, respectively.

The space between the two neighboring shells in a MWCNT is $0.34 \mathrm{~nm}[13,18]$. Thus assuming the diameter of each shell is much larger than $0.34 \mathrm{~nm}$ where this fact is correct for the two neighboring outer shells in a MWCNT, the circuit model parameters which are diameter dependent can be assumed the same for the two neighboring shells. Therefore, in addition to the interaction between outer shells of two adjacent MWCNTs, Figure 5 can be considered for the interaction between the two neighboring outer shells in a MWCNT, with a good approximation.

For calculating the input-output transfer function of the configuration in Figure 5, we need to derivate the total transmission parameter matrix. The ABCD transmission parameter matrix for a uniform $R L C$ transmission line of length $\ell$ is given by [22-25]

$$
T_{R L C}=\left[\begin{array}{cc}
\cosh (\theta \ell) & Z_{0} \sinh (\theta \ell) \\
\frac{1}{Z_{0}} \sinh (\theta \ell) & \cosh (\theta \ell)
\end{array}\right]
$$

where $Z_{0}=\sqrt{(R+s L) /(s C)}, \theta=\sqrt{(R+s L) s C}$, and $s=j \omega$ is the complex frequency. Recall that each segment in Figure 5 is a $R L G C$ model, and the new parameters $Z_{0}$ and $\theta$ should be determined. Figure 6 shows two deferent segments, one is related to the $R L C$ transmission line modeled interconnect, and the other is related to the $R L G C$ transmission line modeled interconnect, respectively.

Comparison of the two sections (a) and (b) of Figure 6 implies that the ABCD transmission parameter matrix for a uniform $R L G C$ transmission line of length $\ell$ can be written as

$$
T_{R L G C}=\left[\begin{array}{cc}
\cosh \left(\ell \theta_{R L G C}\right) & Z_{0, R L G C} \sinh \left(\ell \theta_{R L G C}\right) \\
\frac{1}{Z_{0, R L G C}} \sinh \left(\ell \theta_{R L G C}\right) & \cosh \left(\ell \theta_{R L G C}\right)
\end{array}\right],
$$

where $R_{\mathrm{ex}}=\left(R_{C}+R_{F}\right) / 2$ and

$$
\begin{aligned}
Z_{0, R L G C} & =\sqrt{\left[R_{S}+s(L+k M)\right] /\left[G_{\mathrm{TV}}+s\left(C+C_{\mathrm{CV}}\right)\right]}, \\
\theta_{R L G C} & =\sqrt{\left[R_{S}+s(L+k M)\right]\left[G_{\mathrm{TV}}+s\left(C+C_{\mathrm{CV}}\right)\right]} .
\end{aligned}
$$

The total ABCD transmission parameter matrix of the configuration in Figure 5 can be expressed as

$$
T_{\text {total }}=\left[\begin{array}{cc}
1 & R_{t r} \\
0 & 1
\end{array}\right]\left[\begin{array}{cc}
1 & 0 \\
s C_{\text {out }} & 1
\end{array}\right]\left[\begin{array}{cc}
1 & R_{\mathrm{ex}} \\
0 & 1
\end{array}\right] T_{R L G C}\left[\begin{array}{cc}
1 & R_{\mathrm{ex}} \\
0 & 1
\end{array}\right]
$$

Therefore, using (12) and (14), the matrix $T_{\text {total }}$ can be written as

$$
T_{\text {total }}=\left[\begin{array}{cc}
A_{R L G C} & B_{R L G C} \\
C_{R L G C} & D_{R L G C}
\end{array}\right]
$$

where

$$
\begin{aligned}
A_{R L G C}= & \left(1+s R_{t r} C_{\text {out }}\right) \cosh \left(\ell \theta_{R L G C}\right) \\
& +\frac{\left(R_{t r}+R_{\mathrm{ex}}+s R_{t r} R_{\mathrm{ex}} C_{\text {out }}\right)}{Z_{0, R L G C}} \sinh \left(\ell \theta_{R L G C}\right), \\
B_{R L G C}= & {\left[R_{t r}+2 R_{\mathrm{ex}}+2 s R_{t r} R_{\mathrm{ex}} C_{\mathrm{out}}\right] \cosh \left(\ell \theta_{R L G C}\right) }
\end{aligned}
$$




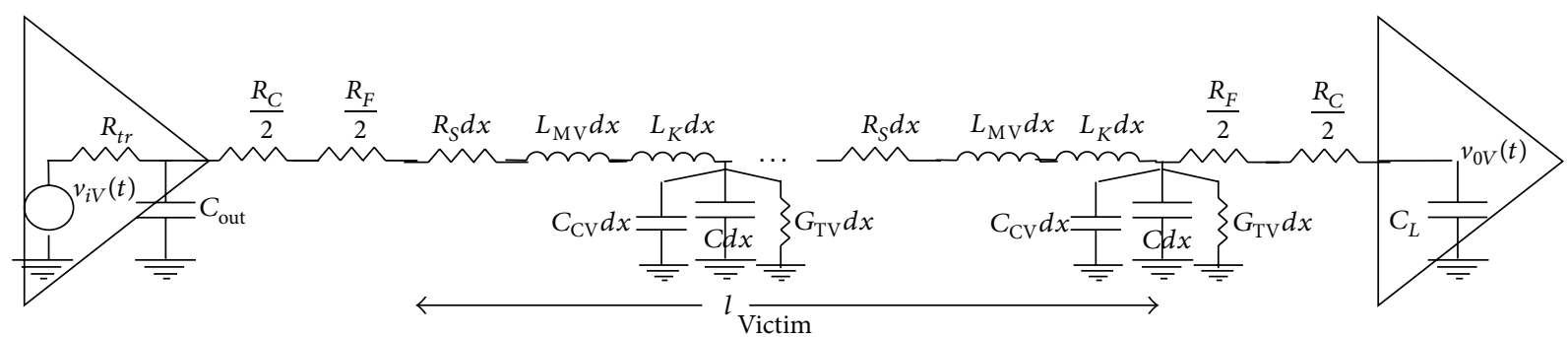

FIGURE 5: Equivalent circuit model for a driven victim CNT interconnect (an outer shell or inner shell of a MWCNT) as a victim line, with considering the impact of aggressor line, using transmission line modeling.

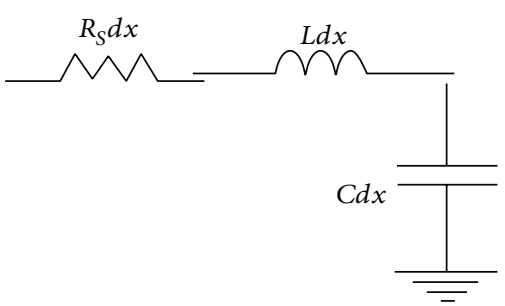

(a)

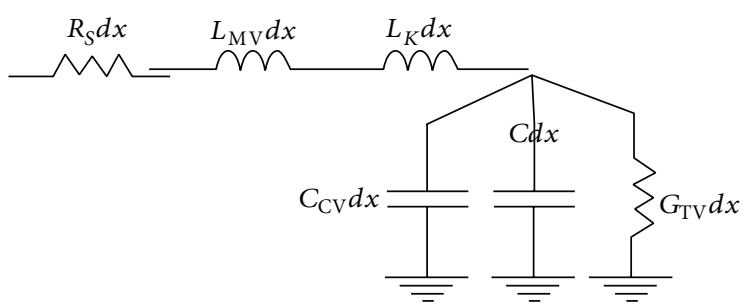

(b)

FIGURE 6: Segments of a transmission line modeled interconnect: (a) RLC model (b) RLGC model.

$$
\begin{aligned}
+ & {\left[Z_{0}\left(1+s R_{t r} C_{\text {out }}\right)\right.} \\
& \left.+\frac{R_{\mathrm{ex}}\left(R_{t r}+R_{\mathrm{ex}}+s R_{t r} R_{\mathrm{ex}} C_{\mathrm{out}}\right)}{Z_{0, R L G C}}\right] \\
& \times \sinh \left(\ell \theta_{R L G C}\right), \\
C_{R L G C}= & s C_{\text {out }} \cosh \left(\ell \theta_{R L G C}\right)+\frac{1+s R_{\mathrm{ex}} C_{\mathrm{out}}}{Z_{0, R L G C}} \sinh \left(\ell \theta_{R L G C}\right), \\
D_{R L G C}= & \left(1+2 s R_{\mathrm{ex}} C_{\text {out }}\right) \cosh \left(\ell \theta_{R L G C}\right) \\
+ & {\left[s C_{\mathrm{out}} Z_{0, R L G C}\right.} \\
& \left.+\frac{R_{\mathrm{ex}}\left(1+s R_{\mathrm{ex}} C_{\mathrm{out}}\right)}{Z_{0, R L G C}}\right] \\
\times & \sinh \left(\ell \theta_{R L G C}\right) .
\end{aligned}
$$

The input-output ratio for the configuration of Figure 5 in Laplace domain can be written as

$$
H(s)=\frac{V_{0 V}(s)}{V_{i V}(s)}=\frac{1}{A_{R L G C}+s C_{L} B_{R L G C}} .
$$

Substituting (16) and (17) in (20) satisfies

$$
H(s)=\left(\left[1+s\left(R_{t r} C_{\text {out }}+R_{t r} C_{L}+2 R_{\mathrm{ex}} C_{L}\right)\right.\right.
$$

$$
\begin{aligned}
& \left.+s^{2}\left(2 R_{t r} R_{\mathrm{ex}} C_{\text {out }} C_{L}\right)\right] \cosh \left(\ell \theta_{\text {RLGC }}\right) \\
& +\left[\frac{R_{t r}+R_{\mathrm{ex}}}{Z_{0, R L G C}}+s\right. \\
& \quad \times\left(\frac{R_{t r} R_{\mathrm{ex}} C_{\text {out }}+R_{\mathrm{ex}}\left(R_{t r}+R_{\mathrm{ex}}\right) C_{L}}{Z_{0, R L G C}}+Z_{0}^{T} C_{L}\right) \\
& \left.\quad+s^{2}\left(\frac{R_{t r} R_{\mathrm{ex}}^{2} C_{\text {out }}}{Z_{0, R L G C}}+Z_{0, R L G C} R_{t r} C_{\text {out }}\right) C_{L}\right] \\
& \left.\times \sinh \left(\ell \theta_{R L G C}\right)\right)^{-1} .
\end{aligned}
$$

For simulation and analysis purposes, we need to convert (21) to a linear parametric relation. As it is clear from (13), if we could neglect the tunneling conductance $G_{\mathrm{TV}}$, then the configuration of Figure 5 would be considered as a distributed (transmission) line $R L C$ circuit where its $R, L$, and $C$ values are $R_{S},\left(L_{\mathrm{MV}}+L_{K}\right)$ and $\left(\mathrm{C}_{\mathrm{CV}}+C\right)$ where $L_{\mathrm{MV}}$ and $C_{\mathrm{CV}}$ are defined in (8) and (10). For this purpose as mentioned in Section 1, two important interaction effects in MWCNT structures might be considered. One effect is between the two neighboring shells in a MWCNT which we call it here "Intertalk effect," and the other is between the two adjacent MWCNTs which we call it here "Crosstalk Effect." As it is clear from Figure 2, for the two coupled neighboring shells, the electron tunneling happens through the outer surface of inner shell. Thus in this case that is corresponding to "Intertalk effect," the tunneling conductance $G_{\mathrm{TV}}$ plays a major role and has a large value, where this fact is compatible with the experimental results [13]. For the case of two coupled adjacent 
MWCNTs as it is clear from Figure 2, the area through which the electron tunneling happens is a narrow longitudinal band between the two MWCNTs. Therefore, in this case that is corresponding to "Crosstalk effect," the tunneling conductance $G_{\mathrm{TV}}$ has no major role and can be neglected in the related computations. This fact is also compatible with the discussion of Section 2.1.2.

In [25], a similar configuration such as Figure 5 for a CNT-bundle interconnect has been discussed and the related input-output transfer function such as (21) has been obtained. In this discussion where no coupling effect has been considered, the CNT-bundle has been modeled as a uniform transmission line $R L C$ circuit and a linear parametric relation for its input-output ratio has been calculated. As mentioned above for the case of "Crosstalk effect" between the two coupled adjacent MWCNTs, we can ignore the tunneling conductance $G_{\mathrm{TV}}$ and therefore Figure 5 can be considered as a transmission line $R L C$. In this case, the inductance and capacitance of each section will be equal to $\left(C+C_{\mathrm{CV}}\right) d x$ and $\left(L_{\mathrm{MV}}+L_{K}\right) d x$ where $L_{\mathrm{MV}}$ and $C_{\mathrm{CV}}$ are defined as (8) and (10), respectively,

$$
\begin{aligned}
& H(s)=\frac{1}{1+A_{1} s+A_{2} s^{2}+A_{3} s^{3}+A_{4} s^{4}+A_{5} s^{5}+A_{6} s^{6}}, \\
& A_{1}=R_{\text {tr }}\left[C_{\text {out }}+\left(C+C_{\mathrm{CV}}\right) \ell+C_{L}\right] \\
& +R_{\mathrm{ex}}\left[\left(C+C_{\mathrm{CV}}\right) \ell+2 C_{L}\right]+R_{S} \ell \\
& \times\left[\frac{\left(C+C_{\mathrm{CV}}\right) \ell}{2 !}+C_{L}\right] \text {, } \\
& A_{2}=R_{\mathrm{ex}} R_{t r}\left(C_{\text {out }}\left(C+C_{\mathrm{CV}}\right) \ell\right. \\
& \left.+C_{L}\left(C+C_{\mathrm{CV}}\right) \ell+2 C_{\text {out }} C_{L}\right) \\
& +R_{t r} R_{S}\left(C+C_{\mathrm{CV}}\right) \ell^{2} \\
& \times\left[\frac{C_{\text {out }}\left(C+C_{\mathrm{CV}}\right) \ell}{2 !}+\frac{\left(C+C_{\mathrm{CV}}\right)^{2} \ell^{2}}{3 !}\right. \\
& \left.+C_{\text {out }} C_{L}+\frac{\left(C+C_{\mathrm{CV}}\right) \ell C_{L}}{2 !}\right] \\
& +R_{\mathrm{ex}} R_{S}\left(C+C_{\mathrm{CV}}\right) \ell^{2} \\
& \times\left[\frac{\left(C+C_{\mathrm{CV}}\right) \ell}{3 !}+C_{L}\right]+\frac{R_{S}^{2}\left(C+C_{\mathrm{CV}}\right)^{2} \ell^{4}}{4 !} \\
& +\frac{(L-k M)\left(C+C_{\mathrm{CV}}\right) \ell^{2}}{2 !}+R_{\mathrm{ex}}^{2}\left(C+C_{\mathrm{CV}}\right) \ell C_{L} \\
& +\frac{R_{S}^{2}\left(C+C_{\mathrm{CV}}\right) \ell^{3} C_{L}}{3 !}+(L-k M) \ell C_{L} \\
& A_{3}=\frac{2 R_{S}(L-k M)\left(C+C_{\mathrm{CV}}\right)^{2} \ell^{4}}{4 !}+\frac{R_{S}^{3}\left(C+C_{\mathrm{CV}}\right)^{3} \ell^{6}}{6 !} \\
& +\left[R_{\mathrm{ex}}+R_{t r}+\frac{R_{S}}{\left(C+C_{\mathrm{CV}}\right)} C_{L}\right]
\end{aligned}
$$

$$
\begin{aligned}
& \times\left[\frac{(L-k M)\left(C+C_{\mathrm{CV}}\right)^{2} \ell^{3}}{3 !}+\frac{R_{S}^{2}\left(C+C_{\mathrm{CV}}\right)^{3} \ell^{5}}{5 !}\right] \\
& +\left[R_{t r}\left(C_{\text {out }}+C_{L}\right)+2 R_{\mathrm{ex}} C_{L}\right] \\
& \times\left[\frac{(L-k M)\left(C+C_{\mathrm{CV}}\right) \ell^{2}}{2 !}+\frac{R_{S}^{2}\left(C+C_{\mathrm{CV}}\right)^{2} \ell^{4}}{4 !}\right] \\
& +\frac{R_{S}\left(C+C_{\mathrm{CV}}\right)^{2} \ell^{3}}{3 !} \times\left[R_{\mathrm{ex}} R_{t r}\left(C_{\mathrm{out}}+C_{L}\right)\right. \\
& +R_{\mathrm{ex}}^{2} C_{L}+\frac{(L-k M)}{\left(C+C_{\mathrm{CV}}\right)} C_{L} \\
& \left.+\frac{R_{S}}{\left(C+C_{\mathrm{CV}}\right)} R_{t r} C_{\text {out }} C_{L}\right] \\
& +R_{\mathrm{ex}} R_{t r} C_{\text {out }}\left(C+C_{\mathrm{CV}}\right) \ell C_{L}\left(R_{\mathrm{ex}}+R_{S} \ell\right) \\
& +R_{t r} C_{\text {out }}(L-k M) \ell C_{L} \text {, } \\
& A_{4}=\frac{(L-k M)^{2}\left(C+C_{\mathrm{CV}}\right)^{2} \ell^{4}}{4 !} \\
& +\frac{3 R_{S}^{2}(L-k M)\left(C+C_{\mathrm{CV}}\right)^{3} \ell^{6}}{6 !} \\
& +\frac{R_{S}^{4}\left(C+C_{\mathrm{CV}}\right)^{4} \ell^{8}}{8 !}+\left[R_{t r}\left(C_{\text {out }}+C_{L}\right)+2 R_{\mathrm{ex}} C_{L}\right] \\
& \times\left[\frac{2 R_{S}(L-k M)\left(C+C_{\mathrm{CV}}\right)^{2} \ell^{4}}{4 !}\right. \\
& \left.+\frac{R_{S}^{3}\left(C+C_{\mathrm{CV}}\right)^{3} \ell^{6}}{6 !}\right] \\
& +\left[R_{t r}+R_{\mathrm{ex}}+\frac{R_{S}}{\left(C+C_{\mathrm{CV}}\right)} C_{L}\right] \\
& \times\left[\frac{2 R_{S}(L-k M)\left(C+C_{\mathrm{CV}}\right)^{3} \ell^{5}}{5 !}\right. \\
& \left.+\frac{R_{S}^{3}\left(C+C_{\mathrm{CV}}\right)^{4} \ell^{7}}{7 !}\right]+2 R_{\text {tr }} R_{\mathrm{ex}} C_{\text {out }} C_{L} \\
& \times\left[\frac{(L-k M)\left(C+C_{\mathrm{CV}}\right) \ell^{2}}{2 !}+\frac{R_{S}^{2}\left(C+C_{\mathrm{CV}}\right)^{2} \ell^{4}}{4 !}\right] \\
& +\left[R_{t r} R_{\text {ex }}\left(C_{\text {out }}+C_{L}\right)+R_{\mathrm{ex}}^{2} C_{L}+\frac{(L-k M)}{\left(C+C_{\mathrm{CV}}\right)} C_{L}\right. \\
& \left.+\frac{R_{S}}{\left(C+C_{\mathrm{CV}}\right)} R_{t r} C_{\text {out }} C_{L}\right] \\
& \times\left[\frac{(L-k M)\left(C+C_{\mathrm{CV}}\right)^{2} \ell^{3}}{3 !}+\frac{R_{S}^{2}\left(C+C_{\mathrm{CV}}\right)^{3} \ell^{5}}{5 !}\right]
\end{aligned}
$$




$$
\begin{aligned}
& +\frac{R_{S}\left(C+C_{\mathrm{CV}}\right)^{2} \ell^{3}}{3 !}\left[R_{t r} R_{\mathrm{ex}}^{2} C_{\mathrm{out}} C_{L}+\frac{(L-k M)}{\left(C+C_{\mathrm{CV}}\right)}\right. \\
& \left.\times R_{t r} C_{\text {out }} C_{L}\right] \\
& A_{5}=\left[R_{t r}\left(C_{\text {out }}+C_{L}\right)+2 R_{\text {ex }} C_{L}\right] \\
& \times\left[\frac{(L-k M)^{2}\left(C+C_{\mathrm{CV}}\right)^{2} \ell^{4}}{4 !}\right. \\
& +\frac{3 R_{S}^{2}(L-k M)\left(C+C_{\mathrm{CV}}\right)^{3} \ell^{6}}{6 !} \\
& \left.+\frac{R_{S}^{4}\left(C+C_{\mathrm{CV}}\right)^{4} \ell^{8}}{8 !}\right]+2 R_{t r} R_{\mathrm{ex}} C_{\text {out }} C_{L} \\
& \times\left[\frac{2 R_{S}(L-k M)\left(C+C_{\mathrm{CV}}\right)^{2} \ell^{4}}{4 !}\right. \\
& \left.+\frac{R_{S}^{3}\left(C+C_{\mathrm{CV}}\right)^{3} \ell^{6}}{6 !}\right] \\
& +\left[R_{t r} R_{\text {ex }}\left(C_{\text {out }}+C_{L}\right)+R_{\text {ex }}^{2} C_{L}\right. \\
& \left.+\frac{(L-k M)}{\left(C+C_{\mathrm{CV}}\right)} C_{L}+\frac{R_{S}}{\left(C+C_{\mathrm{CV}}\right)} R_{t r} C_{\text {out }} C_{L}\right] \\
& \times\left[\frac{2 R_{S}(L-k M)\left(C+C_{\mathrm{CV}}\right)^{3} \ell^{5}}{5 !}\right. \\
& \left.+\frac{R_{S}^{3}\left(C+C_{\mathrm{CV}}\right)^{4} \ell^{7}}{7 !}\right] \\
& +\left[R_{t r} R_{\mathrm{ex}}^{2} C_{\text {out }} C_{L}+\frac{(L-k M)}{\left(C+C_{\mathrm{CV}}\right)} R_{t r} C_{\text {out }} C_{L}\right] \\
& \times\left[\frac{(L-k M)\left(C+C_{\mathrm{CV}}\right)^{2} \ell^{3}}{3 !}\right. \\
& \left.+\frac{R_{S}^{2}\left(C+C_{\mathrm{CV}}\right)^{3} \ell^{5}}{5 !}\right] \\
& A_{6}=2 R_{\text {tr }} R_{\text {ex }} C_{\text {out }} C_{L} \\
& \times\left[\frac{(L-k M)^{2}\left(C+C_{\mathrm{CV}}\right)^{2} \ell^{4}}{4 !}\right. \\
& +\frac{3 R_{S}^{2}(L-k M)\left(C+C_{\mathrm{CV}}\right)^{3} \ell^{6}}{6 !} \\
& \left.+\frac{R_{S}^{4}\left(C+C_{\mathrm{CV}}\right)^{4} \ell^{8}}{8 !}\right]
\end{aligned}
$$

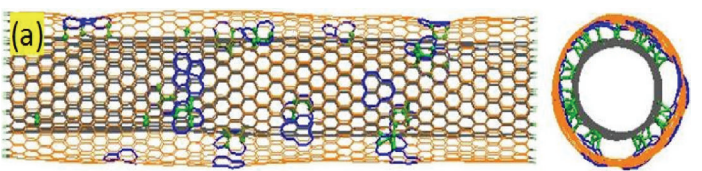

Figure 7: Side and axial view of a $(17,0) /(26,0)$ DWCNT with 20 vacancy defects [29].

$$
\begin{gathered}
+\left[R_{t r} R_{\mathrm{ex}}^{2} C_{\text {out }} C_{L}+\frac{(L-k M)}{\left(C+C_{\mathrm{CV}}\right)} R_{\text {tr }} C_{\text {out }} C_{L}\right] \\
\times\left[\frac{2 R_{S}(L-k M)\left(C+C_{\mathrm{CV}}\right)^{3} \ell^{5}}{5 !}\right. \\
\left.+\frac{R_{S}^{3}\left(C+C_{\mathrm{CV}}\right)^{4} \ell^{7}}{7 !}\right] .
\end{gathered}
$$

2.3. The Analysis of Defective Coupled CNTs. Different structures of carbon nanotubes are usually under various defects such as tensile and compressive behavior and vacancy defects, which have been studied widely by the researchers [26-29]. In the case that the coupled SWCNTs or the walls in a MWCNT are defective or some structural defects are inserted between the CNTs, the behavior of total structure would be complicated. Since the basis of this paper discussion is according to the circuit modeling, the model of modified defective structure needs more attention to be introduced. For this purpose, accurate simulating methods such as ab initio and molecular dynamics should be applied in order to analyze the overall behavior of structure.

In [26], a deformed SWCNT under the tensile strain has been analyzed using Green's function method and a relation for the transmission function has been obtained. In [27], a circuit model for the deformation region of a deformed SWCNT under the tensile strain has been proposed. In order to consider the influence of strain in our analysis for two coupled CNTs or two shells in a MWCNT, one can insert the equivalent circuit model introduced in [27], $L_{D} C_{D}$, between each segment of transmission line in Figures 3 and 5. In [28], the load transfer between the inner and outer nanotubes in a MWCNT has been discussed and analyzed both in tension and compression, using molecular dynamics simulation.

The structural defects usually reduce the load carrying capacity of materials. In [29], an anomalous vacancy-defectinduced enhancement of interwall load transfer in annealed defective carbon nanotubes (CNTs) has been studied and simulated using molecular dynamics method. Figure 7 shows a double-walled carbon nanotube (DWCNT) in which a deformation with 20 vacancy defects has been considered.

The structural defects are usually unwanted and unordered throughout the SWCNTs or MWCNTs; therefore, proposing a circuit model such as Figures 3 and 5 would be imprecise and complicated. For obtaining reasonable and accurate results in this respect, as mentioned above, the analysis methods such as molecular dynamics and $a b$ 


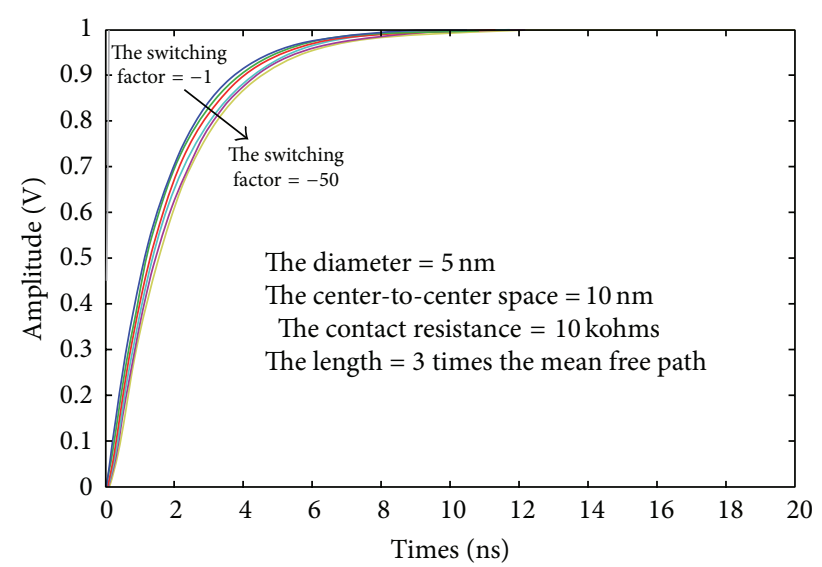

$\begin{array}{ll}\text { The switching factor }=-1 & \text { The switching factor }=-30 \\ \text { The switching factor }=-10 & \text { The switching factor }=-40 \\ \text { The switching factor }=-20 & \text { The switching factor }=-50\end{array}$

FIGURE 8: The output response of the configuration in Figure 5 to the ramp input with the slope of $10 \mathrm{~V} / \mathrm{ns}$, using (22), for various values of the switching factor.

initio which consider the atomic and subatomic calculations should be adopted.

\section{Simulation Results}

Figure 8 shows the output response of the configuration in Figure 5 to the ramp input with the slope of $10 \mathrm{~V} / \mathrm{ns}$, using (22), for various values of the switching factor from -50 to -1 . The output resistance and capacitance of the drivers have been assumed as $80 \Omega$ and $12 \mathrm{fF}$, respectively. Also the load capacitance has been chosen $45 \mathrm{fF}$. Recall that $\lambda_{\mathrm{CNT}} \approx 1000 \mathrm{D}$ [13], where $D$ is the diameter of CNT, the mean free path of each CNT $\left(\lambda_{\mathrm{CNT}}\right)$ has been considered $5 \mu \mathrm{m}$.

As it is clear from Figure 8, the propagation delay at the end of victim line increases with the increase of absolute switching factor. These variations will be discussed in the next section.

3.1. The Crosstalk-Induced Delay. Figure 9 shows the crosstalk-induced delay at the end of victim line (the configuration of Figure 5), for various values of the switching factor from -50 to -1 . In this figure, the length of each CNT has been chosen 3 times the mean free path, and therefore as discussed in the previous section for the mean free path of $5 \mu \mathrm{m}$, the length value will be $15 \mu \mathrm{m}$.

As shown in Figure 9, for the length of coupled CNTs equal to $15 \mu \mathrm{m}$, the crosstalk-induced delay changes from $1.16 \mathrm{~ns}$ to $1.56 \mathrm{~ns}$ for the absolute switching factor values from 1 to 50 , that is, a variation range of 34.5 percent.

3.2. The Impact of Contact Resistance. In [14], the impact of contact resistance on the behavior of CNT bundles in VLSI interconnects has been discussed and also a brief discussion of the contact resistance for SWCNTs has been presented. In [16], the electrical contacts to SWCNTs for Rh (Rhodium)

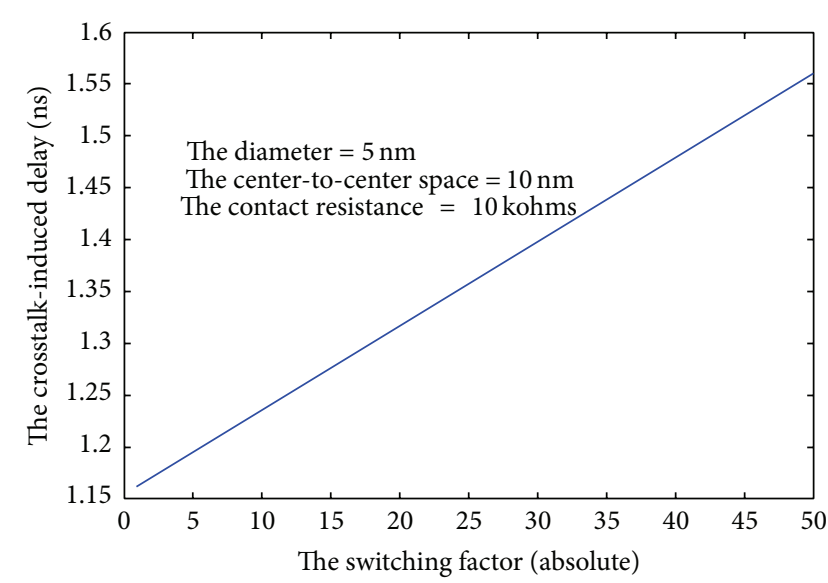

FIGURE 9: The crosstalk-induced delay at the end of victim line (the configuration of Figure 5), for various values of the switching factor.

and Pd (Palladium) contact materials have been studied, and the related contact resistance dependence to the diameter of CNT has been investigated. Based on the experimental measurements done in [16], the following quadratic relation for the normalized increase in contact resistance of a SWCNT versus the diameter, for Pd and Rh contacts, has been suggested in [14]:

$$
D_{\mathrm{rc}}=\frac{D^{2}-2.811 D+2.538}{0.5376 D^{2}-0.8106 D+0.3934}
$$

where $D_{\mathrm{rc}}$ and $D$ are the normalized increase in contact resistance and the diameter of CNT, respectively. The contact resistance remains relatively constant for $D>2 \mathrm{~nm}$ and (23) is valid for $1 \leq D \leq 2 \mathrm{~nm}[14,16]$. Also no measurements have been performed for $D<1 \mathrm{~nm}$, although the obtained results show the increase of contact resistance with the decrease of diameter.

Figure 10 shows the output response of the configuration in Figure 5 to the ramp input with the slope of $10 \mathrm{~V} / \mathrm{ns}$, using (22), for various values of the contact resistance from 0 to 50 $\mathrm{k} \Omega$. In this figure, the switching factor $k$ has been assumed equal to -1 .

\section{Conclusions}

In this paper, we have studied the crosstalk effects between the two coupled SWCNTs (the outer shells of two adjacent MWCNTs) and the intertalk effects between the two neighboring shells in a MWCNT. One of the two coupled CNTs has been defined as the victim line and the other as the aggressor line. Using the transmission line circuit modeling, a new parametric transfer function has been obtained for the analysis of the impact of aggressor line on the victim line. This transfer function depends on various coupling parameters such as the mutual inductance, the coupling capacitance, and the tunneling resistance. Using the obtained transfer function, the time behavior of the coupling effects due to the aggressor line on the victim line and an important effect named as "crosstalk-induced delay" have been analyzed. The 


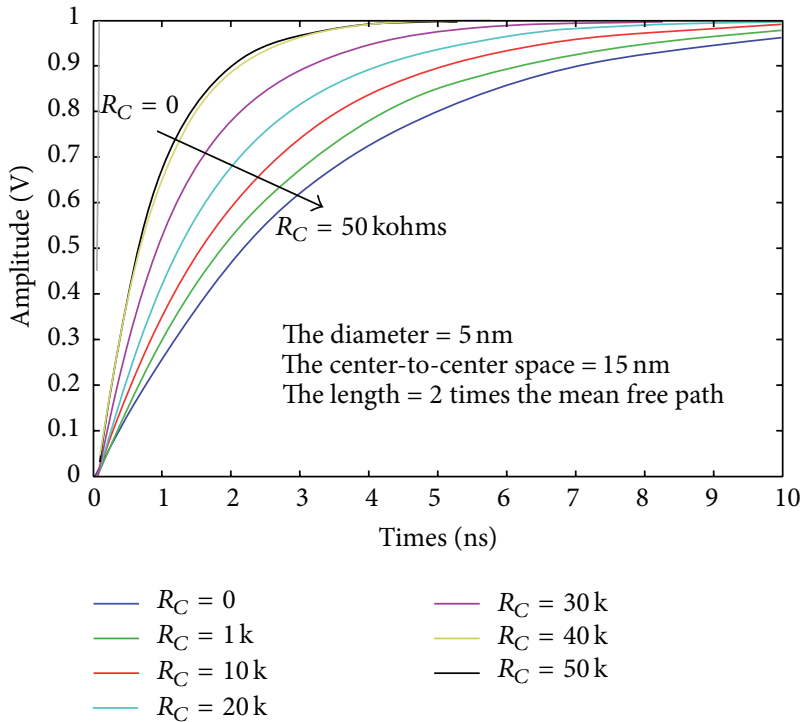

Figure 10: The output response of the configuration in Figure 5 to the ramp input with the slope of $10 \mathrm{~V} / \mathrm{ns}$, using (22), for various values of the contact resistance and with the switching factor $k=-1$.

impact of contact resistance variations on the time behavior of coupling effects and also the impact of switching factor changing on the crosstalk-induced delay have been studied and analyzed. Also in this study, the influence of defects on the behavior of defective coupled CNTs has been discussed in short.

\section{Conflict of Interests}

The author declares that there is no conflict of interests regarding the publication of this paper.

\section{References}

[1] Y. Xu and A. Srivastava, "A model for Carbon Nanotube Interconnects," International Journal of Circuit Theory and Applications, vol. 38, no. 6, pp. 559-575, 2010.

[2] C. T. White and T. N. Todorov, "Quantum electronics: Nanotubes go ballistic," Nature, vol. 411, no. 6838, pp. 649-651, 2001.

[3] F. Davood and B. Forouzandeh, "A novel approach for stability analysis in carbon nanotube interconnects," IEEE Electron Device Letters, vol. 30, no. 5, pp. 475-477, 2009.

[4] A. Naeemi, R. Sarvari, and J. D. Meindl, "Performance comparison between carbon nanotube and copper interconnects for gigascale integration (GSI)," IEEE Electron Device Letters, vol. 26, no. 2, pp. 84-86, 2005.

[5] J. Li, Q. Ye, A. Cassell et al., "Bottom-up approach for carbon nanotubes interconnects," Applied Physics Letters, vol. 82, pp. 2491-2493, 2003.

[6] S. Datta, "Electrical resistance: an atomistic view," Nanotechnology, vol. 15, pp. S433-S451, 2004.

[7] S. Datta, Electronic Transport in Mesoscopic Systems, Cambridge University Press, New York, NY, USA, 1995.

[8] N. Srivastava and K. Banerjee, "Performance analysis of carbon nanotube interconnects for VLSI applications," in Proceedings of the IEEE/ACM International Conference on Computer-Aided Design (ICCAD '05), pp. 383-390, San Jose, Calif, USA, November 2005.

[9] K. Banerjee and N. Srivastava, "Are carbon nanotubes the future of VLSI interconnections?" in Proceedings of the ACM Design Automation Conference (DAC '06), pp. 809-814, San Francisco, Calif, USA, July 2006.

[10] P. J. Burke, "Luttinger liquid theory as a model of the gigahertz electrical properties of carbon nanotubes," IEEE Transactions on Nanotechnology, vol. 1, no. 3, pp. 129-144, 2002.

[11] A. Raychowdhury and K. Roy, "Modeling of metallic carbonnanotube interconnects for circuit simulations and a comparison with $\mathrm{Cu}$ interconnects for scaled technologies," IEEE Transactions on Computer-Aided Design of Integrated Circuits and Systems, vol. 25, no. 1, pp. 58-65, 2006.

[12] P. J. Burke, "An RF circuit model for carbon nanotubes," IEEE Transactions on Nanotechnology, vol. 2, no. 1, pp. 55-58, 2003.

[13] H. Li, W. Y. Yin, K. Banerjee, and J. F. Mao, "Circuit modeling and performance analysis of multi-walled carbon nanotube interconnects," IEEE Transactions on Electron Devices, vol. 55, no. 6, pp. 1328-1337, 2008.

[14] A. Nieuwoudt and Y. Massoud, "Evaluating the impact of resistance in carbon nanotube bundles for VLSI interconnect using diameter-dependent modeling techniques," IEEE Transactions on Electron Devices, vol. 53, no. 10, pp. 2460-2466, 2006.

[15] A. Nieuwoudt and Y. Massoud, "Understanding the impact of inductance in carbon nanotube bundles for VLSI interconnect using scalable modeling techniques," IEEE Transactions on Nanotechnology, vol. 5, no. 6, pp. 758-764, 2006.

[16] W. Kim, A. Javey, R. Tu, J. Cao, Q. Wang, and H. Dai, "Electrical contacts to carbon nanotubes down to $1 \mathrm{~nm}$ in diameter," Applied Physics Letters, vol. 87, no. 17, Article ID 173101, 2005.

[17] A. Raychowdhury and K. Roy, "A circuit model for carbon nanotube interconnects: comparative study with $\mathrm{Cu}$ interconnects for scaled technologies," in Proceedings of the IEEE/ACM International Conference on Computer-Aided Design (ICCAD '04), pp. 237-240, November 2004.

[18] M. F. Yu, B. I. Yakobson, and R. S. Ruoff, "Controlled sliding and pullout of nested shells in individual multiwalled carbon nanotubes," Journal of Physical Chemistry B, vol. 104, no. 37, pp. 8764-8767, 2000.

[19] B. Bourlon, C. Miko, L. Forró, D. C. Glattli, and A. Bachtold, "Determination of the intershell conductance in multiwalled carbon nanotubes," Physical Review Letters, vol. 93, no. 17, pp. 176806/1-176806/4, 2004.

[20] A. Stetter, J. Vancea, and C. H. Back, "Determination of the intershell conductance in a multiwall carbon nanotube," Applied Physics Letters, vol. 93, no. 17, Article ID 172103, 2008.

[21] C. Patrick Yue and S. Simon Wong, "Physical modeling of spiral inductors on silicon," IEEE Transactions on Electron Devices, vol. 47, no. 3, pp. 560-568, 2000.

[22] K. Banerjee and A. Mehrotra, "Analysis of on-chip inductance effects for distributed RLC interconnects," IEEE Transactions on Computer-Aided Design of Integrated Circuits and Systems, vol. 21, no. 8, pp. 904-915, 2002.

[23] A. K. Palit, W. Anheier, and J. Schloeffel, "Reduced order long interconnect modeling," 15. ITG-GI-GMM Workshop Testmethoden und Zuverlässigkeit von Schaltungen und Systemen, Timmendorfer Strand, pp. 42-47, 2003.

[24] D. Fathi and B. Forouzandeh, "Time domain analysis of carbon nanotube interconnects based on distributed RLC model," Nano, vol. 4, no. 1, pp. 13-21, 2009. 
[25] D. Fathi, B. Forouzandeh, S. Mohajerzadeh, and R. Sarvari, "Accurate analysis of carbon nanotube interconnects using transmission line model," Micro and Nano Letters, vol. 4, no. 2, pp. 116-121, 2009.

[26] D. Fathi, B. Forouzandeh, and R. Sarvari, "A new method for the analysis of transmission property in carbon nanotubes using Green's function," Applied Physics A, vol. 102, no. 1, pp. 231-238, 2011.

[27] D. Fathi and R. Sarvari, "A new model for deformed carbon nanotubes using Green's function," Applied Physics A, vol. 105, no. 4, pp. 875-880, 2011.

[28] G. A. Shen, S. Namilae, and N. Chandra, "Load transfer issues in the tensile and compressive behavior of multiwall carbon nanotubes," Materials Science and Engineering A, vol. 429, no. 1-2, pp. 66-73, 2006.

[29] D. Santo Pietro, C. Tang, and C. Chen, "Enhancing interwall load transfer by vacancy defects in carbon nanotubes," Applied Physics Letters, vol. 100, no. 3, Article ID 033118, 2012. 

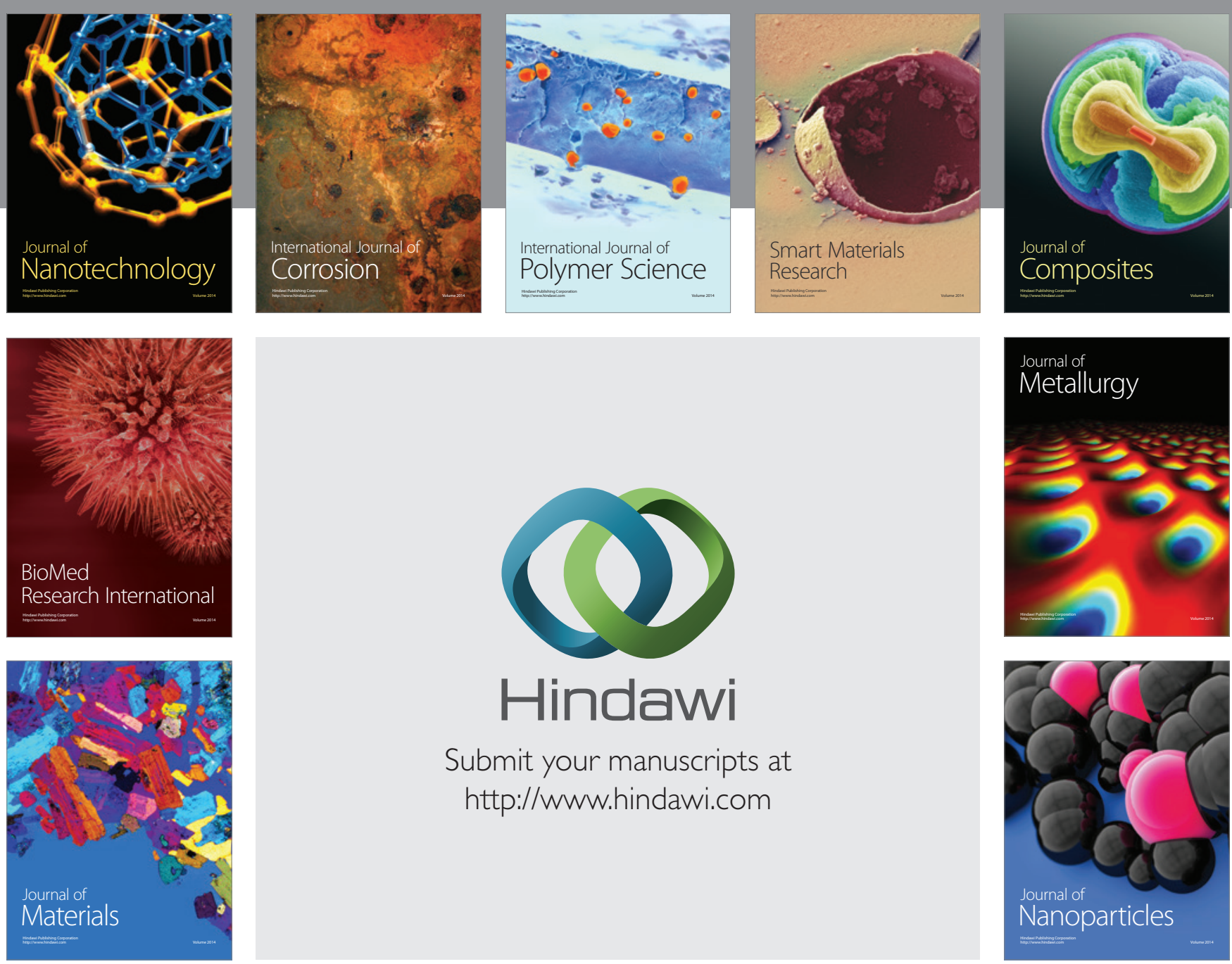

Submit your manuscripts at http://www.hindawi.com
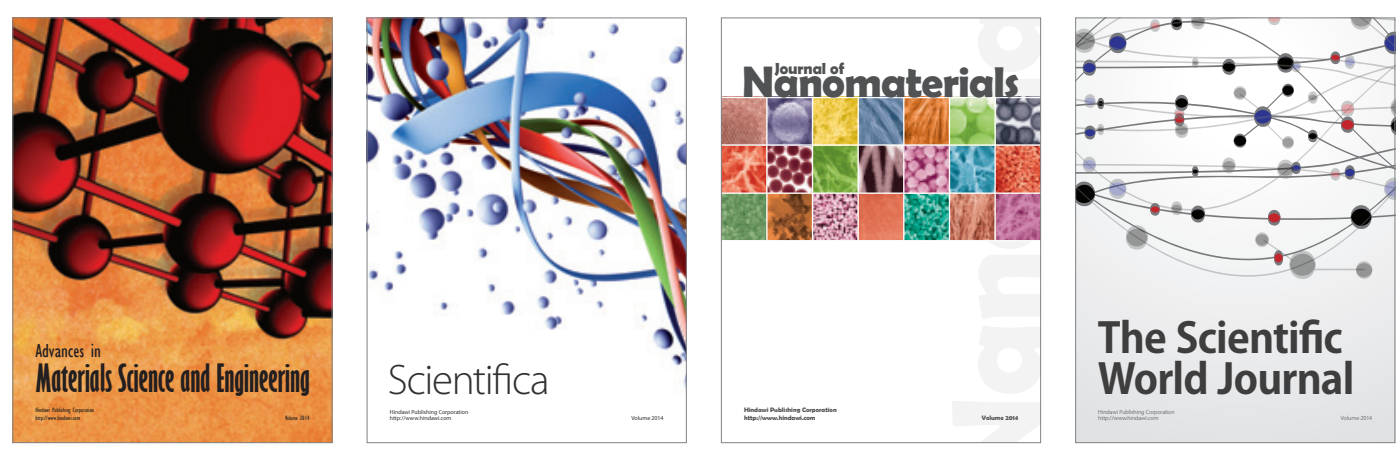

\section{The Scientific World Journal}
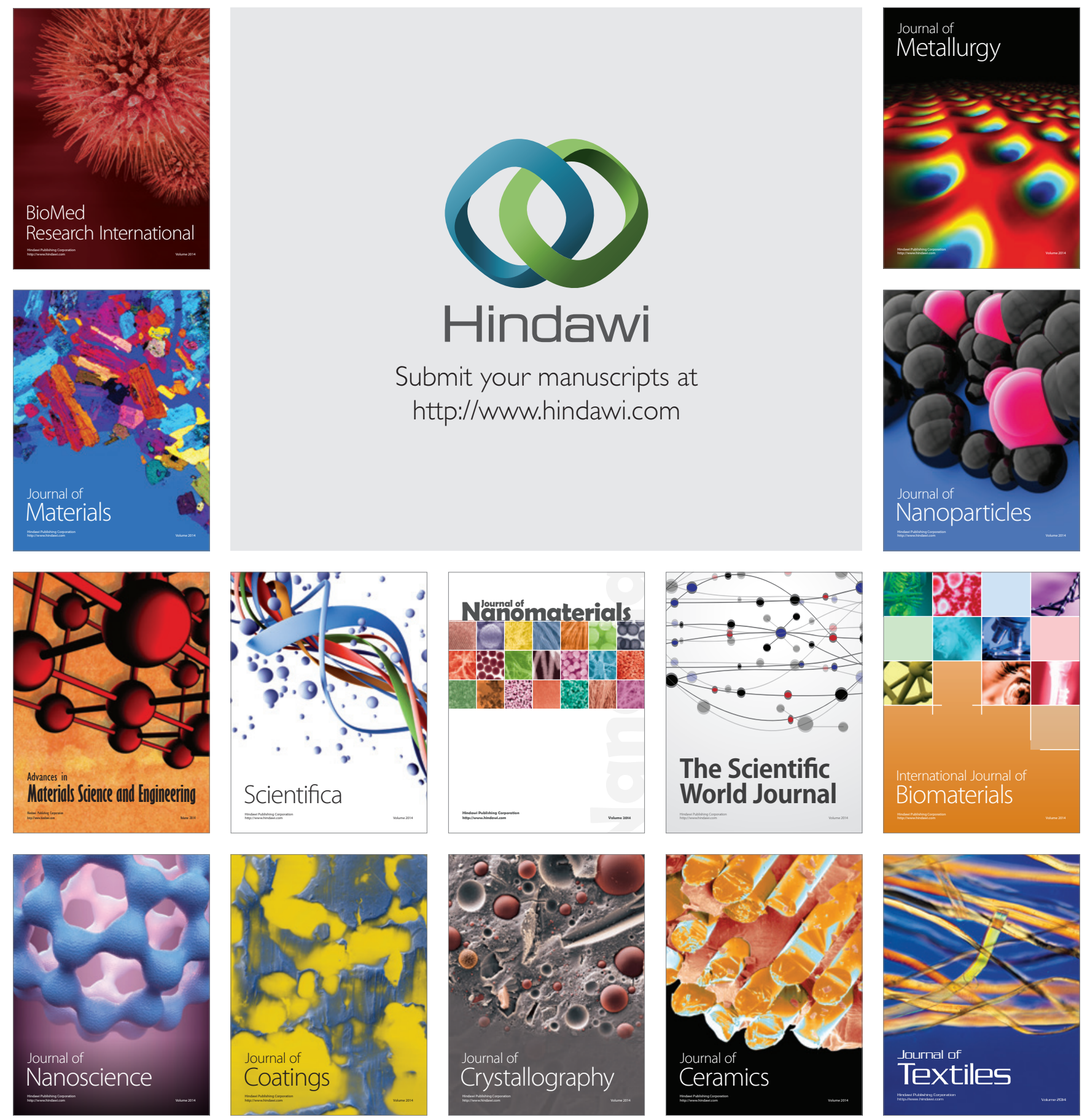